\title{
Expression of CA125 and cisplatin susceptibility of pleural effusion-derived human lung cancer cells from a Thai patient
}

\author{
PITHI CHANVORACHOTE ${ }^{1}$, SUDJIT LUANPITPONG $^{2}$, PREEDAKORN CHUNHACHA ${ }^{2}$, \\ WORRAWAT PROMDEN ${ }^{3}$ and VIROTE SRIURANPONG ${ }^{4}$ \\ ${ }^{1}$ Department of Pharmacology and Physiology; ${ }^{2}$ Pharmaceutical Technology (International) Program; \\ ${ }^{3}$ Department of Pharmacognosy and Pharmaceutical Botany, Faculty of Pharmaceutical Sciences, \\ Chulalongkorn University; ${ }^{4}$ Division of Medical Oncology, Department of Medicine, Faculty of Medicine, \\ Chulalongkorn University and the King Chulalongkorn Memorial Hospital, Bangkok, Thailand
}

Received January 20, 2012; Accepted May 4, 2012

DOI: $10.3892 / \mathrm{ol} .2012 .711$

\begin{abstract}
Advances in understanding lung cancer biology and tumor markers aid clinicians in managing the disease. Cancer-associated antigen (CA)125 has garnered increasing attention in lung cancer research and may benefit the treatment and follow-up of this type of cancer. In Thai lung cancer patients, knowledge regarding ethnic differences in cancer cell biology is largely absent. We generated lung cancer cells from the pleural effusion fluids of a Thai patient and designated these as P1 cells. P1 cells were assessed for growth rate, response to chemotherapy, and the presence of tumor markers, in particular CA125 expression. Results of immunofluorescence indicated that $\mathrm{P} 1$ cells exhibited strong expression levels of CA125, comparable to that of established H460 lung cancer cells. Furthermore, P1 cells were analyzed for the expression of additional markers. Results revealed that H460 cells exhibited strong immunofluorescent signals from cytokeratin-19 fragments (CYFRA 21-1) and squamous cell carcinoma antigen (SCCA) while P1 presented only CYFRA 21-1 signals. We also found evidence of relative cisplatin resistance in P1 compared to the susceptibility level of established lung cancer cells. Thus, the results and methodology described in this study may aid the development of lung cancer diagnostic and therapeutic approaches and, in particular, advance understanding of ethnic differences.
\end{abstract}

\section{Introduction}

The identification of tumor markers leads to a significant improvement in cancer therapy and aids investigators in understanding cancer biology (1). Tumor markers are useful

Correspondence to: Dr Pithi Chanvorachote, Department of Pharmacology and Physiology, Faculty of Pharmaceutical Sciences, Chulalongkorn University, Phatumwan, Bangkok 10330, Thailand E-mail: pithi_chan@yahoo.com; pithi.c@chula.ac.th

Key words: Thai, lung cancer, cancer-associated antigen 125 , cisplatin in cancer detection and classification (2). Moreover, in treating patients, serum levels of such tumor markers are helpful in the determination of cancer prognosis and likelihood of recurrence (3). Among several well-known tumor markers, cancer-associated antigen (CA)125 has garnered increasing attention in lung cancer research. CA125 is a glycoprotein found on the cell membrane which has been used as a standard tumor marker for the diagnosis and follow-up of ovarian cancers $(4,5)$. Moreover, a significant increase of CA125 has been reported in other cancers including breast and lung cancers has been noted $(6,7)$.

Among the various anti-cancer agents used for lung cancer treatment, cisplatin [cis-diamminedichloroplatinum (II)] is commonly prescribed (8). Cisplatin mediates cancer cell apoptosis through reactive oxygen species (ROS)-dependent and DNA adduct pathways $(9,10)$. Cisplatin-induced apoptosis is mainly mediated through the caspase signaling pathway (11). Although available lung cancer cell lines have been widely used for the investigation of cancer cell biology and chemotherapeutic susceptibility, none of the cell lines were from Thai patients. Since ethnic diversity is known to be a factor affecting tumor marker expression and chemotherapeutic response $(12,13)$ and knowledge regarding such cancer signatures of Thai-originated cancer cells remains elusive, the present study aimed to generate knowledge about the tumor marker expression and chemotherapeutic response of lung cancer cells obtained from a Thai patient. Consequently, we generated primary cancer cells collected from the pleural effusion fluid of a Thai patient and characterized the lung cancer signatures of the cells compared to the established lung cancer H460 cells. The obtained cells were evaluated for CA125 expression and response to cisplatin, one of the most widely used chemotherapeutic agents. The results and methodology described in this study may aid the development of lung cancer diagnostic and therapeutic approaches and, in particular, advance understanding of ethnic differences.

\section{Materials and methods}

Clinical specimens and reagents. Pleural effusions were collected from a 76-year-old male Thai patient with suspected 
lung adenocarcinoma. Informed consent was obtained from the patient and the study was approved by the ethics committee of the Faculty of Medicine and the ethics committee of the faculty of Pharmaceutical Sciences, Chulalongkorn University. Human proximal tubular epithelial renal cells (HK-2) and human lung cancer epithelial (H460) cells were obtained from the American Type Culture Collection (Manassas, VA, USA). H460 cells were cultured in RPMI-1640 while HK-2 cells were cultured in DMEM, supplemented with $10 \%$ fetal bovine serum (FBS), 2 mM L-glutamine and $100 \mathrm{U} / \mathrm{ml}$ penicillin/ streptomycin in a $5 \% \mathrm{CO}_{2}$ environment at $37^{\circ} \mathrm{C}$. Cisplatin, propidium iodide (PI) and Hoechst 33342 were obtained from Sigma Chemical, Inc. (St. Louis, MO, USA). Resazurin was purchased from Invitrogen (Carlsbad, CA, USA). Specific antibodies for CA125, CYFRA 21-1, and SCCA were obtained from Santa Cruz Biotechnology (Santa Cruz, CA, USA). Resazurin and Alexa Fluor 488 goat anti-rabbit IgG $(\mathrm{H}+\mathrm{L})$ and Alexa Fluor 590 goat anti-mouse IgG $(\mathrm{H}+\mathrm{L})$ conjugated secondary antibody were purchased from Invitrogen.

Specimen preparation. Pleural effusion was centrifuged at $1600 \mathrm{x} \mathrm{g}$ for $10 \mathrm{~min}$ at room temperature. The pellet was resuspended with $4 \mathrm{ml}$ sterile balanced salt solution and then centrifuged on a Ficoll gradient (Ficoll-Paque ${ }^{\mathrm{TM}}$, GE Healthcare, Piscataway, NJ, USA) at $400 \mathrm{x}$ g for $40 \mathrm{~min}$ at $20^{\circ} \mathrm{C}$ to separate the tumor cells from erythrocytes. The layer of mononuclear cells were collected and washed twice with 3 volumes of RPMI-1640 by centrifugation at $400 \mathrm{x}$ g for $10 \mathrm{~min}$ at $20^{\circ} \mathrm{C}$. The pellet was then resuspended and the cells were cultured in ACL-4 medium supplemented with $5 \% \mathrm{FBS}$ at $37^{\circ} \mathrm{C}$ and $5 \% \mathrm{CO}_{2}$.

Growth properties and cell morphology. Cells were initially seeded at a density of $1 \times 10^{4}$ cells in a 24 -well plate and the population doubling time was determined. At various time points, cells were trypsinized by $0.25 \%$ trypsin-EDTA treatment and the number of cells was calculated by the trypan blue exclusion method. Population doubling times were determined from an exponential regression of viable cell counts over nine days.

Immunofluorescence. Cells (5x104/well) were seeded in 6-well plates for $24 \mathrm{~h}$ to allow the cells to completely adhere to the surface. The cells were then fixed in $3.7 \%$ formaldehyde for $10 \mathrm{~min}$ at room temperature, permeabilized and blocked in a solution containing $0.5 \%$ saponin, $1 \%$ FBS and $1.5 \%$ goat serum for $30 \mathrm{~min}$. Following primary antibody incubation with CA125, CYFRA 21-1 or SCCA antibody at 1:100 dilution for $1 \mathrm{~h}$, cells were washed and incubated together with Alexa Fluor 488 goat anti-rabbit $\mathrm{IgG}(\mathrm{H}+\mathrm{L})$-conjugated secondary antibody (Invitrogen) or Alexa Fluor 590 goat anti-mouse IgG $(\mathrm{H}+\mathrm{L})$ conjugated secondary antibody (Invitrogen) for $30 \mathrm{~min}$. Nuclei were stained with Hoechst dye (Invitrogen). Images were visualized by fluorescence microscopy (Olympus IX51 with DP70).

Cytotoxicity and apoptosis assay. Cytotoxicity was determined by a Presto Blue fluorescence assay. Following specific treatments, cells in a 96-well plate were incubated with 1:50 resazurin for $1 \mathrm{~h}$ at $37^{\circ} \mathrm{C}$. Fluorescence intensity of resazurin product (resorufin) was measured at $530 \mathrm{~nm}$ (excitation wavelength) and $590 \mathrm{~nm}$ (emission wavelength) using a microplate
A

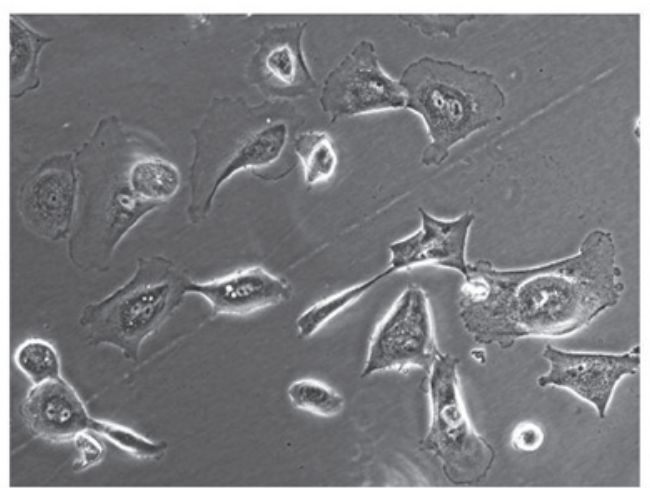

B

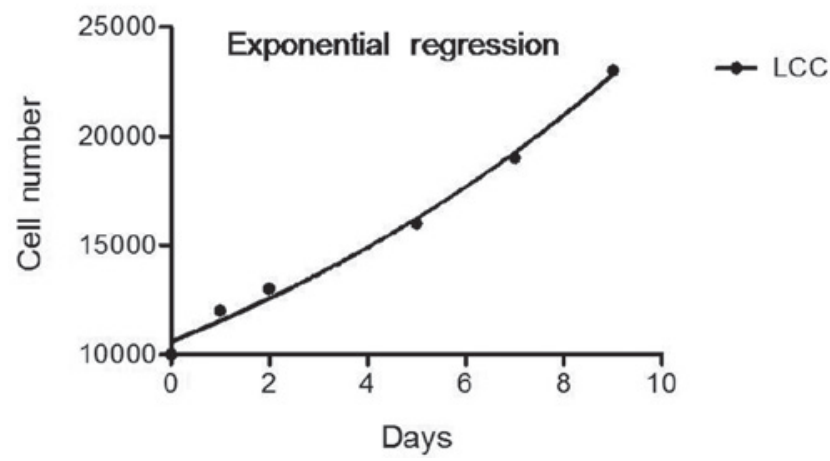

Figure 1. Phase-contrast micrographs showing morphology of a pleural effusion-derived lung carcinoma cells. (A) P1 cells exhibited epitheloid morphology with a bipolar, spindle-shaped appearance. (B) Population doubling time of $\mathrm{P} 1$ cells were calculated based on the exponential regression of cell growth.

reader. Cell viability was calculated as a percentage relative to non-treated cells. Analyses were performed independently in triplicate. Apoptosis was determined by a Hoechst 33342 DNA fragmentation assay. Briefly, cells were incubated with $10 \mu \mathrm{g} /$ $\mathrm{ml}$ of Hoechst 33342 for $30 \mathrm{~min}$ and analyzed for apoptosis by scoring the percentage of cells having intensely condensed chromatin and/or fragmented nuclei on fluorescence microscopy (Olympus IX51 with DP70). The apoptotic index was calculated as the percentage of cells with apoptotic nuclei over the total number of cells.

Statistical analysis. The data are presented as the means \pm SD from three or more independent experiments. Statistical analysis was performed using the Student's t-test at a significance level of $\mathrm{P}<0.05$.

\section{Results}

Growth properties and morphology of lung carcinoma cells. Pleural effusion fluids of an untreated Thai patient were first centrifuged and the suspended cells were collected. The cells obtained were cultured under adherent conditions in lung cancer cell-selective ACL-4 medium as described by the National Cancer Institute (NCI) for the selective growth of non-small cell lung cancer, while rarely sparing the growth of normal cells, such as fibroblasts and macrophages. In the present study, those cells continuously propagating for at least 
A

Phase-Hoechst
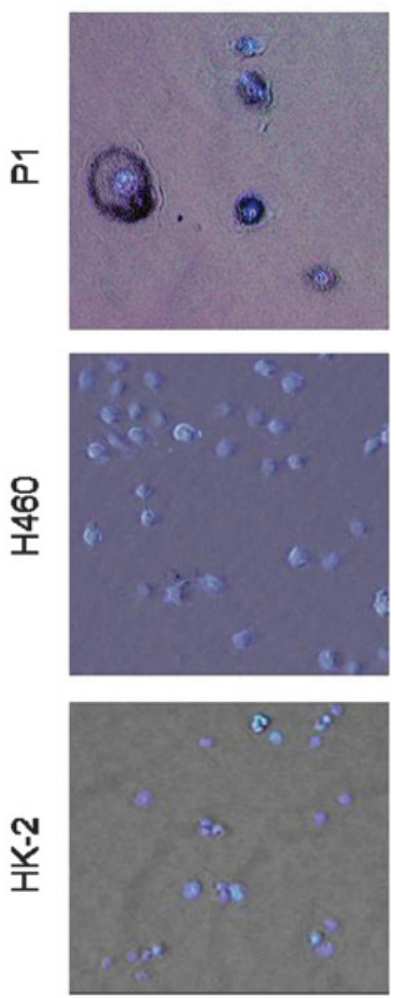

CA125
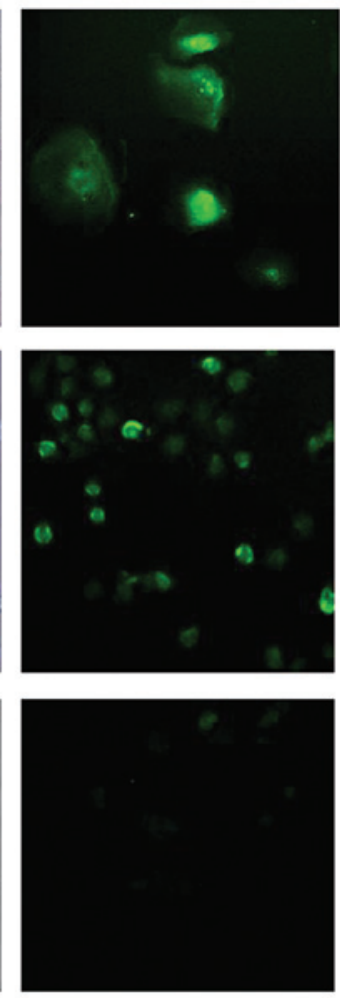

B

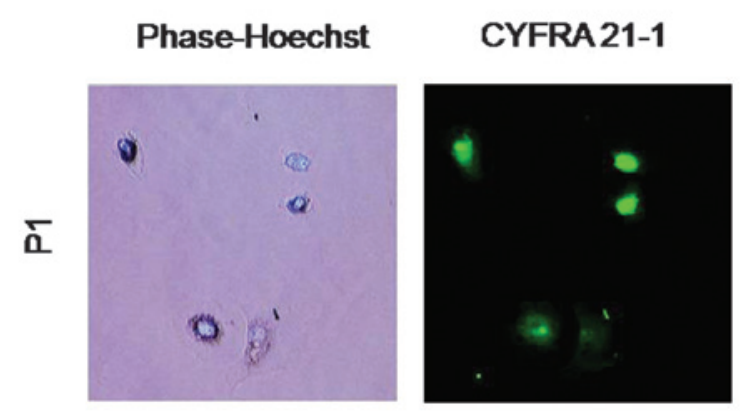

Phase-Hoechst

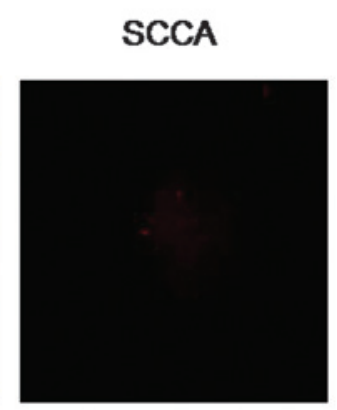

Figure 2. Immunocytochemistry evaluation for CA125, CYFRA 21-1 and SCCA expression. Primary lung carcinoma P1, lung cancer H460, and proximal epithelial HK-2 cells were evaluated for CA125 expression. The expression of CYFRA 21-1 and SCCA in P1 cells was evaluated by immunofluorescence as described in Materials and methods. The fluorescence signal was detected under a fluorescence microscope.

six months were designated as P1 cells. The clinical and pathological details of the patient from whom P1 cells were obtained are described in Materials and methods. Six months later, cells were routinely cultured in ACL-4 medium supplemented with $10 \%$ FBS. P1 cells exhibited epitheloid morphology with a bipolar, spindle-shaped appearance (Fig. 1A). Cells grew individually with little cell-cell contact. This morphology of P1 cells was markedly similar to the previously established nonsmall cell lung cancer cell lines from solid tumor specimens (14). Population doubling time of P1 cells was calculated based on the exponential regression of cell growth (Fig. 1B). The best-fit population doubling value was 8.127 days. Although the cells were slow-growing, they could be maintained in ACL-4 medium and continue to be cultured for a long period of time.

Expression of CA125 in Pl cells. Recent evidence suggests that CA125 may be an important tumor marker for lung cancer. Data obtained from the present study revealed that the expression profile of primary lung cancer cells originating from a Thai patient confirms this hypothesis and may facilitate the development of a lung cancer marker; we therefore evaluated the expression of CA125 in P1 cells. Immunohistochemical investigation using anti-CA125 antibody indicated that CA125 was highly expressed in P1 cells as in the positive control lung carcinoma H460 cells. Notably, CA125 signals originated from the nuclear region of the cells as well as the cell surface (Fig. 2A). Moreover, we evaluated the specificity of CA125 expression on tumor cells by staining the non-cancerous proximal tubule epithelial HK-2 cells. Results indicated that even though HK-2 cells were stained by the CA125 antibody, the signal was only slightly increased (Fig. 2A). To provide supporting evidence for the histological sub-type of P1 cells, we performed an additional immunofluorescence study using CYFRA 21-1 (a marker for non-small cell carcinoma) and anti-SCCA (a marker for squamous cell carcinoma) antibodies. P1 cells were found to express CYFRA 21-1 but not SCCA protein (Fig. 2B). The results indicated that P1 may be either adenocarcinoma or large cell carcinoma, but not squamous carcinoma.

Cisplatin response of $P 1$ cells. Disruption of apoptosis contributes to malignant cell growth and chemotherapeutic resistance. To determine the apoptosis phenotype of P1 cells, the cells were treated with various concentrations of cisplatin (0-200 $\mu \mathrm{M})$ and analyzed for cell toxicity by resazurin assay and apoptosis by Hoechst 33342 assay. H460 cells were used as a standard phenotype for non-small cell lung cancer. Cells having intensely condensed and/or fragmented nuclei were considered apoptotic. Treatment with cisplatin induced a dose-dependent decrease in cell viability and a concomitant increase of apoptotic cells in P1 and H460 cells (Fig. 3). $\mathrm{P} 1$ cells acquired higher apoptosis resistance to cisplatin as compared to H460 cells (Fig. 3B). We summarized the $\mathrm{IC}_{50}$ values of $\mathrm{P} 1, \mathrm{H} 460$, and the additional A549 lung cancer cell line in Table I. Since apoptotic resistance is a key 


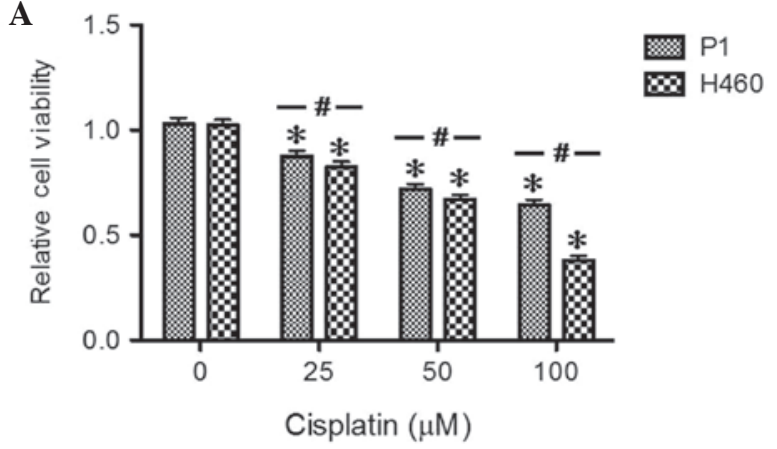

B
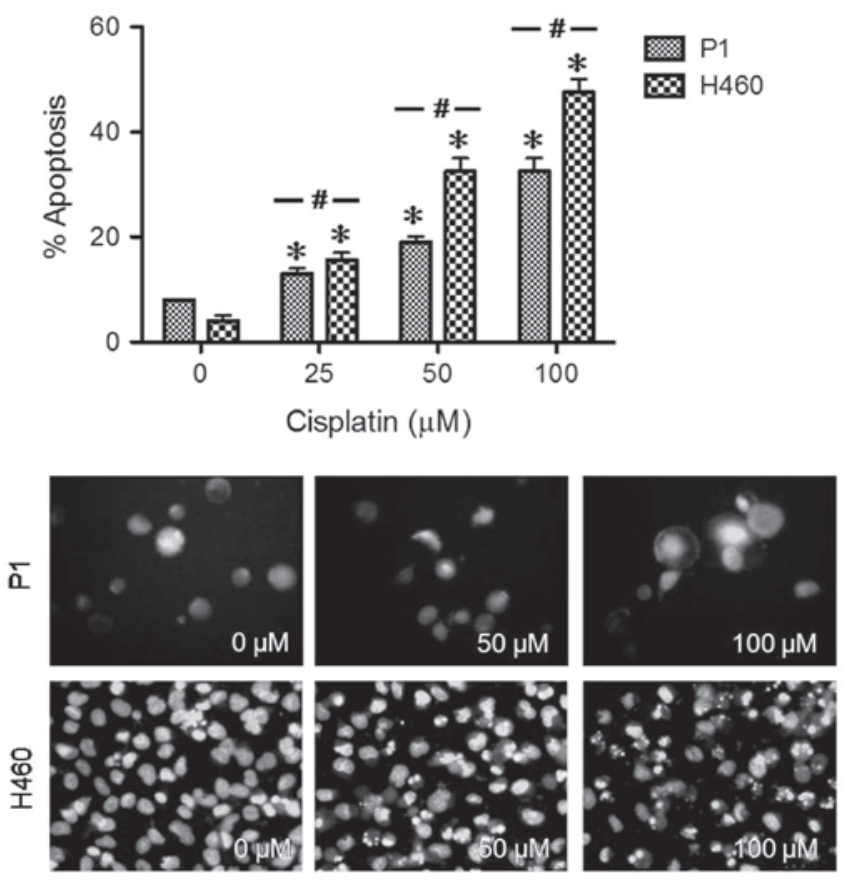

Figure 3. Susceptibility of cells in response to cisplatin-mediated apoptosis. Primary lung carcinoma P1 and lung cancer H460 cells were evaluated for their apoptotic response to various concentrations of cisplatin $(0-100 \mu \mathrm{M})$. Cell viability and apoptosis were evaluated by resazurin and Hoechst 33342 assays, respectively. Values are the means \pm SD $(n=3)$.

characteristic of all cancer cells, this finding suggested the lung cancer-like signature of P1 cells.

\section{Discussion}

Improved understanding of cancer cell biology is likely to benefit the overall improvement of cancer therapy. In Thailand, the number of diagnosed lung cancer patients is on the increase and has become a primary cause of cancer-related mortality (15).Satisfactory clinical outcomes for lung cancer treatment are currently limited by two main obstacles which include late detection and the presence of chemotherapeutic resistance (16). Overcoming these barriers is vital, with improvement of basic knowledge of lung cancer biology, tumor markers and cellular response to chemotherapeutic agents being crucial. The present study generated P1 cells from the pleural effusion of a Thai male who was diagnosed as a lung cancer patient. The cells demonstrated typical cancerous characteristics including CYFRA 21-1 expression.
Table I. Comparison of cisplatin susceptibility of cells.

\begin{tabular}{lc}
\hline Cells & $\mathrm{IC}_{50}(\mu \mathrm{M})$ \\
\hline H460 & $80 \pm 8.61$ \\
P1 & $151 \pm 10.51$ \\
A549 & $62 \pm 5.32$ \\
\hline
\end{tabular}

P1, H460, and A549 lung cancer cells were incubated with various concentrations of cisplatin for $24 \mathrm{~h}$. The reduction of cell viability was determined by a resazurin viability assay and the $\mathrm{IC}_{50}$ values were then calculated. Values are the means $\pm \operatorname{SD}(n=3)$.

CA125 expression in lung cancer cells has previously been reported and the accumulated data suggested that this tumor marker may be of significance for lung cancer therapy (6). Furthermore, CA125 expression in lung cancer patients may be a good predictive tool for patient outcome (17). CA125 is an important tumor marker recognized by a monoclonal antibody OC125. CA125 is elevated in the serum of ovarian cancer patients and has been identified as a useful tool for the screening of ovarian cancer $(4,5,18)$. In lung cancer, investigators have found a significant expression of CA125 in a number of lung cancer cell lines (7). In accordance with these findings, we found a strong expression of such tumor markers in lung carcinoma H460 (obtained from ATCC) and Thai-originated lung cancer P1 cells.

Accumulating evidence indicates that ethnic difference is a notable factor in the determination of the chemotherapeutic response. In the case of anti-cancer agents, regimens including doses and drug manipulating protocols are often used for different ethnic populations. In response to chemotherapeutic agents, a number of cell mechanisms are activated, supporting the idea that genetic variation by ethnicity may affect the drug responsiveness. Cisplatin is widely used for the treatment of numerous solid tumors including lung cancers (19). The mechanisms of action of the drug on tumor cells is through ROS production and DNA adduct formation $(9,10)$. To induce cell death, several signaling pathways are activated in the regulation of survival and apoptosis and the variation in genetics may alter the cell response. Variation in response to cisplatin among cells was revealed in the present study. Two lung cancer cell models obtained from ATCC, H460 and A549, cells were used as standard cells in the evaluation of cisplatin susceptibility. Results of viability in response to cisplatin revealed that $\mathrm{P} 1$ exhibited slight resistance to cisplatin-mediated death compared to H460 (Fig. 3) and A549 cells (data not shown). For the apoptotic evaluation, the number of apoptotic cells in response to cisplatin in the $\mathrm{P} 1$ population significantly decreased as compared to those of H460 cells (Fig. 3B), suggesting that $\mathrm{P} 1$ cells exhibited relative cisplatin resistance. Consistent results obtained from further investigation revealed that the cisplatin $\mathrm{IC}_{50}$ of $\mathrm{P} 1, \mathrm{H} 460$, and $\mathrm{A} 549$ cells was $151 \pm 10.51,80 \pm 8.61$, and $62 \pm 5.32 \mu \mathrm{M}$, respectively.

Based on these data, it is possible that the variation in ethnicity plays a significant role in drug susceptibility. However, investigations as to this role are required. The present study 
provides a basis for the better understanding of ethnic difference in cancer cell biology.

\section{Acknowledgements}

This study was supported by the higher education research promotion and national research university project of Thailand, office of the higher education commission and postdoctoral fellowship (Ratchadaphiseksompot Endowment Fund, Chulalongkorn University). The authors would like to thank Mr. Krich Rajprasit, a proofreader.

\section{References}

1. Pamies RJ and Crawford DR: Tumor markers - an update. Med Clin N Am 80: 185-199, 1996.

2. Trape J, Molina R and Sant F: Clinical evaluation of the simultaneous determination of tumor markers in fluid and serum and their ratio in the differential of serous effusions. Tumor Biol 25: 276-281, 2004.

3. Hayes DF, Bast R C, Desch CE, et al: Tumor marker utility grading system: a framework to evaluate clinical utility of tumor markers. J Natl Cancer I 88: 1456-1466, 1996.

4. Zurawski VR Jr, Knapp R C, Einhorn N, et al: An initial analysis of preoperative serum CA 125 levels in patients with early stage ovarian carcinoma. Gynecol Oncol 30: 7-14, 1988.

5. Bast RC Jr, Klul TL and St John E: A radioimmunoassay using a monoclonal antibody to monitor the course of epithelial ovarian cancer. New Engl J Med 309: 883-887, 1983.

6. Kimura Y, Fujii T, Hamamoto K, et al: Serum CA125 level is a good prognostic indicator in lung cancer. Brit J Cancer 62: 676-678, 1990
7. Homma S, Satoh H, Kagohashi K, et al: Production of CA125 by human lung cancer cell lines. Clin Exp Med 4: 139-141, 2004.

8. Arriagada R, Bergman B, Dunant A, et al: Cisplatin-based adjuvant chemotherapy in patients with completely resected non-small-cell lung cancer. New Engl J Med 350: 351-360, 2004.

9. Wang D and Lippard SJ: Cellular processing of platinum anticancer drugs. Nat Rev Drug Discov 4: 307-320, 2005.

10. Wang L, Chanvorachote P, Toledo D, et al: Peroxide is a key mediator of Bcl-2 down-regulation and apoptosis induction by cisplatin in human lung cancer cells. Mol Pharmacol 73: 119-127, 2008.

11. Wu YJ, Muldoon LL and Neuwelt EA: The chemoprotective agent $\mathrm{N}$-acetylcyseine blocks cisplatin-induced apoptosis through caspase signaling pathway. J Pharmacol Exp Ther 312: 424-463, 2005 .

12. Gilliland FD: Ethnic differences in cancer incidence: a marker for inherited susceptibility? Environ Health Persp 105: 897-900, 1997.

13. O'Donnell PH and Dolan ME: Cancer pharmacoethnicity: ethnic differences in susceptibility to the effects of chemotherapy. Clin Cancer Res 15: 4806-4814, 2009.

14. Musuda N, Fukuoka M, Takada M, Kudoh S and Kusunoki Y: Establishment and characterization of 20 human non-small cell lung cancer cell lines in a serum-free defined medium (ACL-4). Chest 100: 429-438, 1991.

15. Vatanasapt V, Sriamporn S and Vatanasapt P: Cancer control in Thailand. Jpn J Clin Oncol 32: S82-S91, 2002.

16. Read C, Janes S, George J and Spiro S: Early lung cancer: screening and detection. Prim Care Respir J 15: 332-336, 2006.

17. Buccheri $G$ and Ferrigno D: Lung tumour markers in oncology practice: a study of TPA and CA125. Brit J Cancer 87: 1112-1118, 2002.

18. Jacobs IJ, Skates SJ, MacDonald N, et al: Screening for ovarian cancer: a pilot randomized controlled trial. Lancet 353 : 1207-1210, 1999

19. Pabla $\mathrm{N}$ and Dong Z: Cisplatin nephrotoxicity: mechanisms and renoprotective strategies. Kidney Int 73: 994-1007, 2008. 